\title{
ON A TAUBERIAN THEOREM OF LANDAU
}

BASIL GORDON

1. Introduction. It was the plan of Tschebyschef [1] and Sylvester [2] to deduce the prime number theorem ${ }^{1}$

$$
\psi(x) \sim x
$$

from the formula

$$
T(x)=\sum_{n \leq x} \psi\left(\frac{x}{n}\right)=\log [x] !
$$

However they succeeded only in proving the existence of positive constants $c_{1}$ and $c_{2}$ such that

$$
c_{1} x<\psi(x)<c_{2} x .
$$

Landau discusses this problem in his Handbuch [3, pp. 79-83], where he proves that if $A(x)$ is any monotone nondecreasing function of $x$ such that

$$
T(x)=\sum_{n \leq x} A\left(\frac{x}{n}\right)=x \log x+b x+o(x)
$$

with $b$ a constant, then

$$
c_{1} x<A(x)<c_{2} x
$$

where $c_{1}$ and $c_{2}$ are positive constants. This gives Tschebyschef's theorem if we take

$$
A(x)=\psi(x)
$$

and use the fact that

$$
\log [x] !=x \log x-x+O(\log x) .
$$

Landau states that if only the condition

$$
T(x)=x \log x+O(x)
$$

is assumed, then (1) does not follow, a remark whose incorrectness was proved by $H$. N. Shapiro [4]. Landau then goes on to prove [3, pp. 598-604] that if

Received by the editors June 14, 1957 and, in revised form, November 25, 1957 and February 24, 1958.

${ }^{1}$ Here and in the sequel, $\psi(x), \wedge(n), \mu(n)$, and $\theta(x)$ denote the usual functions of prime number theory. 


$$
T(x)=x \log x+b x+o\left(\frac{x}{\log ^{2} x}\right),
$$

then $A(x) \sim x$. The proof requires the formula

$$
\sum_{n=1}^{\infty} \frac{\mu(n) \log n}{n}=-1
$$

and since this is "deeper" than the prime number theorem, he concludes that Tschebyschef was foredoomed to failure. In the present paper we shall correct this statement by showing that a slightly stronger theorem than Landau's can be obtained using only the prime number theorem. The theorem to be proved is

Theorem 1. If $A(x)$ is nondecreasing, and

$$
T(x)=x \log x+b x+o\left(\frac{x}{\log x}\right)
$$

then $A(x) \sim x$.

It should be mentioned that the best theorem in this direction has been obtained by A. E. Ingham [5], but the proof makes use of Wiener's Tauberian theorem, and therefore cannot be considered elementary.

2. A Selberg-like formula. We shall require the following elementary lemma:

Lemma 1. If $F(x)$ is any function defined for $x \geqq 1$, and if

$$
G(x)=\log x \sum_{n \leqq x} F\left(\frac{x}{n}\right),
$$

then

$$
F(x) \log x+\sum_{n \leqq x} F\left(\frac{x}{n}\right) \Lambda(n)=\sum_{n \leqq x} \mu(n) G\left(\frac{x}{n}\right) .
$$

The proof is a straight-forward application of the Möbius inversion formula. For details, see Trost [6, p. 66]. Now let $A(x)$ be a function for which the hypothesis (3) is satisfied, and apply Lemma 1 with $F(x)=A(x)$. The corresponding $G(x)$ is

$$
G(x)=\log x \sum_{n \unlhd x} A\left(\frac{x}{n}\right)=T(x) \log x=x(\log x)^{2}+b x \log x+o(x),
$$

and so by Lemma 1 , 


$$
\begin{aligned}
A(x) & \log x+\sum_{n \leq x} A\left(\frac{x}{n}\right) \Lambda(n) \\
& =\sum_{n \leqq x} \mu(n) \frac{x}{n}\left(\log \frac{x}{n}\right)^{2}+\sum_{n \leqq x} \mu(n) b \frac{x}{n} \log \frac{x}{n}+\sum_{n \leqq x} \mu(n) o\left(\frac{x}{n}\right) .
\end{aligned}
$$

It is well known that the right side of (4) equals $2 x \log x+o(x \log x)$ so that we obtain

TheOREM 2. If $A(x)$ satisfies condition (3), then

$$
A(x) \log x+\sum_{n \leqq x} A\left(\frac{x}{n}\right) \Lambda(n)=2 x \log x+o(x \log x) .
$$

It can be seen that this result is very similar to the Selberg formula for $\theta(x)$ with a weaker error term (cf. [7], especially the remark on p. 313).

3. Proof of the main theorem. We shall now derive Theorem 1 from the identity (5), and it is here that the monotonicity of $A(x)$ becomes important. Note first that (5) and the fact that $A(x)$ is nondecreasing imply that $A(x)>0$ for all sufficiently large $x$. This fact together with (5) then yields that for $x=y+o(x)$ we have $A(x)$ $=A(y)+o(x)$. Thus it is easily seen that without loss of generality we may assume that for all $x>0$, we have $A(x)=A([x])$. Since $A(x)$ is nondecreasing we can then write

$$
A(x)=\sum_{m \leqq x} a_{m},
$$

where $a_{m} \geqq 0$. We can then rewrite (5) as

$$
A(x) \log x+\sum_{m n \leq x} a_{m} \Lambda(n)=2 x \log x+o(x \log x),
$$

or equivalently

$$
A(x) \log x+\sum_{m \unlhd x} a_{m} \psi\left(\frac{x}{m}\right)=2 x \log x+o(x \log x) .
$$

From the prime number theorem and the result of [4] (in particular Equation (6) therein) it follows that

$$
\sum_{m \leqq x} a_{m} \psi\left(\frac{x}{m}\right)=(1+o(1)) x \sum_{m \leqq x} \frac{a_{m}}{m} \sim x \log x,
$$

which together with (6) above yields $A(x) \sim x$, the desired result.

The following application of Theorem 1 is interesting. Let $A(x)$ $=[x]+M(x)$, where $M(x)=\sum_{n \unlhd x} \mu(n)$. Since $1+\mu(n) \geqq 0, A(x)$ is 
monotone. The corresponding $T(x)$ is

$$
\begin{aligned}
T(x) & =\sum_{n \unlhd x}\left(\left[\frac{x}{n}\right]+M\left(\frac{x}{n}\right)\right) \\
& =x \log x+c x+O\left(x^{1 / 2}\right)+\sum_{n \leq x} \mu(n)\left[\frac{x}{n}\right] \\
& =x \log x+c x+O\left(x^{1 / 2}\right)
\end{aligned}
$$

since $\sum_{n \leqq x} \mu(n)[x / n]=1$ (cf. [3, pp. 576-577]). Thus the hypothesis of Theorem 1 is satisfied and we conclude that $A(x) \sim x$, which is here the same as asserting $M(x)=o(x)$. The above thus provides another proof of the result of Landau [8] which asserts that $\psi(x) \sim x$ implies $M(x)=o(x)$.

\section{REFERENCES}

1. P. L. 'Tschebyschef, Memoire sur les nombres premiers, J. Math. Pures Appl. series 1 , vol. 17 (1852) pp. 366-390.

2. J. J. Sylvester, On Tschebyschef's theorem of the totality of prime numbers comprised within given limits, Amer. J. Math. vol. 4 (1881) pp. 230-247.

3. E. Landau, Handbuch der Lehre von der Verleilung der Primzahlen, Leipzig, 1909.

4. H. N. Shapiro, On the number of primes less than or equal to $x$, Proc. Amer. Math. Soc. vol. 1 (1950) pp. 346-348.

5. A. E. Ingham, Some Tauberian theorems connected with the prime number theorem, J. London Math. Soc. vol. 20 (1945) pp. 171-180.

6. E. Trost, Primzahlen, Basel, 1953.

7. A. Selberg, An elementary proof of the prime number theorem, Ann. of Math. vol. 50 (1949) pp. 305-313.

8. E. Landau, Über den Zusammenhang einiger neuerer Sätze der analytischen Zahlentheorie, Wiener Sitzungsberichte, Math. Klasse, vol. 115 (1906) pp. 589-632.

9. P. Erdös, On a new method in elementary number theory which leads to an elementary proof of the prime number theorem, Proc. Nat. Acad. Sci. U.S.A. vol. 35 (1949) pp. 374-384.

California Institute of Technology 